\title{
Tebipenem Pivoxil
}

National Cancer Institute

\section{Source}

National Cancer Institute. Tebipenem Pivoxil. NCI Thesaurus. Code C76566.

An orally available pivaloyloxymethyl ester prodrug of tebipenem, a broad-spectrum 1beta-methylcarbapenem antibiotic with a 1-(1,3-thiazolin-2-yl) azetidin-3-ylthio group at the C-2 position. After oral administration of tebipenem pivoxil, the ester bond is cleaved, releasing active tebipenem. 